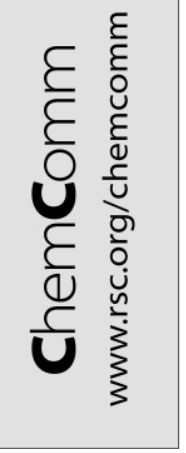

\title{
Bidirectional iterative synthesis of alternating benzene-furan oligomers towards molecular wires $\dagger$
}

\author{
Chin-Fa Lee, ${ }^{a b}$ Ching-Yuan Liu, ${ }^{b}$ Hua-Can Song, ${ }^{a b}$ Shr-Jie Luo, ${ }^{a}$ Jui-Chang Tseng, ${ }^{a b}$ Hsi-Hua Tso ${ }^{a}$ \\ and Tien-Yau Luh*ab \\ a Institute of Chemistry, Academia Sinica, Taipei, Taiwan 115 \\ ${ }^{b}$ Department of Chemistry, National Taiwan University, Taipei, Taiwan 106. \\ E-mail: tyluh@chem.sinica.edu.tw; Fax: +886-2-2651-1488; Tel: +886-2-2789-8500
}

Received (in Cambridge, UK) 12th August 2002, Accepted 10th October 2002

First published as an Advance Article on the web 28th October 2002

Reaction of propargylic dithioacetal 2a with BuLi gives the sulfur-substituted allenyllithium $3 \mathrm{a}$ which is allowed to react with a dialdehyde to yield the corresponding alternating benzene-furan oligoaryls 6 . Functional group transformation converts the ester groups in 6 to dialdehyde 8 which can be used for the synthesis of higher homologues towards molecular wires. A combination of this furan annulation, Heck reaction and Sonogashira coupling leads to a variety of benzene-furan-alkene/alkyne conjugated oligomers of precise length.

There has been ever burgeoning study on the design and synthesis of conjugated oligomers of precise length and constitution because of their potential optoelectronic applications. ${ }^{1}$ Oligoaryls and their vinylene or acetylene homologues have been widely investigated. Incorporation of heteroaromatic rings into these systems will tune the optoelectronic properties of the oligomers, and studies on oligothiophenes or pyrroles and related compounds has been extensive. ${ }^{1,2}$ Relatively speaking, investigations on furan-containing oligoaryls have been only sporadically explored. ${ }^{3-5}$ We recently reported a new annulation procedure for the synthesis of 2,3,5-trisubstituted furans from the corresponding dithioacetals (eqn. (1)). ${ }^{6}$ Thermally and

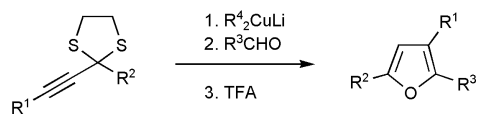

photochemically stable pentaaryls 1 containing alternating benzene and furan moieties are conveniently obtained. The use of these materials as hole transporting materials in electroluminescent devices has been disclosed. ${ }^{7}$ Furthermore, the presence of an alkyl substituent at the $\mathrm{C}-3$ position of the furan heterocycle may increase the solubility of $\mathbf{1}$ in organic solvents. We felt that this protocol can be applied for the bidirectional iterative synthesis of furan-containing oligoaryls of different conjugation lengths leading to molecular wires. Our strategy therefore involves the synthesis of furan-containing oligoaryls functionalized at both terminal aryl moieties.

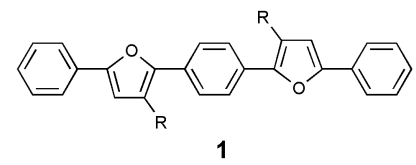

Both organocopper and alkyllithium reagents are known to react with cyclic dithioacetals yielding the corresponding alkylated sulfur-stabilized anions. ${ }^{6,8}$ In order to synthesize the furan-containing oligoaryls functionalized at terminal aryl moieties, it is necessary to test the relative reactivity of different functional groups towards these organometallic reagents. It is known that an ester group can be stable in certain organolithium reagents. ${ }^{9}$ Thus, treatment of $\mathbf{2 a}$ with $\mathrm{BuLi}\left(-78{ }^{\circ} \mathrm{C}, 50 \mathrm{~min}\right)$ yielded 3a (eqn. (2)) which was allowed to react with aldehyde

$†$ Electronic supplementary information (ESI) available: experimental section. See http://www.rsc.org/suppdata/cc/b2/b207881c/

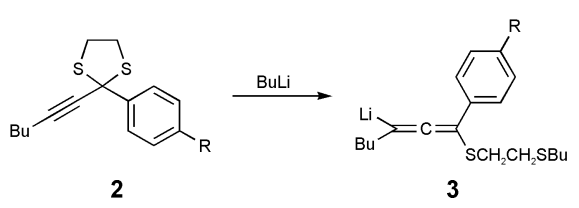

a R $=\mathrm{CO}_{2} \mathrm{Me} \quad \boldsymbol{b} \mathrm{R}=\mathrm{H}_{2} \mathrm{C}=\mathrm{CH} \quad \mathbf{c R}=\mathrm{TMSC} \equiv \mathrm{C} \quad \mathbf{d} \mathrm{R}=\mathrm{H}$

4a $\left(-78{ }^{\circ} \mathrm{C}, 0.5 \mathrm{~h}\right.$, then $\left.\mathrm{rt}, 0.5 \mathrm{~h}\right)$ followed by treatment with TFA (rt, 12 h). After usual work-up, furan-containing diester 5 (mp $110-111^{\circ} \mathrm{C}$ ) was obtained in $67 \%$ yield. $¥$ It is striking to learn that the dithioacetal functionality is more reactive than the ester group towards BuLi under these conditions. This reaction provides a useful route for the synthesis of functionalized allenyllithium reagents by means of a lithium/sulfur exchange reaction. This promising result apparently lays a foundation for the bidirectional iterative synthesis of furan-containing oligoaryls leading to molecular wires.

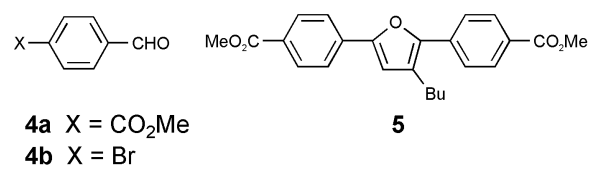

In a similar manner, treatment of 2 equiv. of $\mathbf{3 a}$ with terephthaldehyde $\left(-78{ }^{\circ} \mathrm{C}, 0.5 \mathrm{~h}\right.$, then $\left.\mathrm{rt} 1 \mathrm{~h}\right)$ followed by reaction with TFA (rt, $12 \mathrm{~h}$ ) afforded diester 6a (mp 169-170 ${ }^{\circ} \mathrm{C}$ ) in $45 \%$ yield. Reaction of $6 \mathbf{a}$ with DIBAH (4 equiv., $0{ }^{\circ} \mathrm{C}$. $0.5 \mathrm{~h}$, then $\mathrm{rt} 5 \mathrm{~h}$ ) followed by oxidation with 4 equiv. of $\mathrm{MnO}_{2}$ (rt, $20 \mathrm{~min}$ ) afforded the corresponding dialdehyde 8a $(\mathrm{mp}$ $189-190^{\circ} \mathrm{C}$ ) in $78 \%$ yield. Dialdehyde 8 a was employed for the next annulation reaction with 2.4 equiv. of $\mathbf{3 a}$ to afford the corresponding nonaaryl $\mathbf{6 b}\left(\mathrm{mp} 209-211^{\circ} \mathrm{C}\right)$ in $38 \%$ yield. In a similar manner, $\mathbf{6 b}$ was converted into $\mathbf{8 b}\left(\mathrm{mp} 220-222^{\circ} \mathrm{C}\right)$ in $78 \%$ yield by sequential treatment with DIBAH and $\mathrm{MnO}_{2}$. By employing the same strategy, $13-\operatorname{mer} 6 \mathbf{c}\left(\mathrm{mp} 221-222^{\circ} \mathrm{C}\right)$ was obtained in $32 \%$ yield from $\mathbf{8 b}$ and $\mathbf{3 a}$. Because of the presence of the butyl groups, the solubility of $\mathbf{6}$ in organic solvents was good. They can also easily be precipitated by adding methanol to the organic solutions. Since $\mathbf{6 c}$ contains two ester groups at the terminal phenyl rings, further transformation by repeating the same procedures just mentioned would lead to higher homologues of alternating benzene-furan molecular wires.

Furan-containing oligoaryls 6 were both thermally $\left(T_{\mathrm{d}}=\right.$ $384,410,457{ }^{\circ} \mathrm{C}$ for $\mathbf{6 a}$, b and c, respectively) and photochemically (Sunlamp $200 \mathrm{~W}, 160{ }^{\circ} \mathrm{C}, 24 \mathrm{~h}$ ) stable under

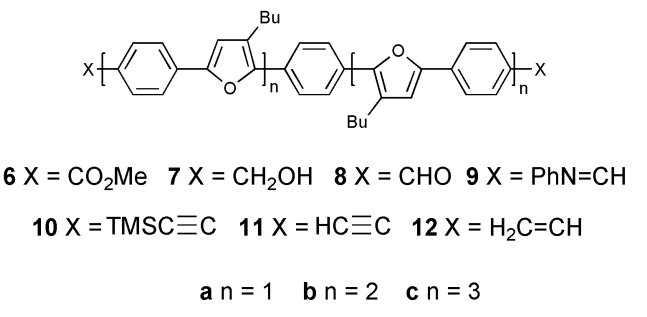


nitrogen atmosphere. However, they underwent decomposition upon irradiation in the presence of air.

A pyrrole moiety can also be introduced into the oligoaryl system when imine was employed as an electrophile. Thus, the reaction of diimine $9 \mathrm{a}$ with $\mathbf{3 d}$ at $-78{ }^{\circ} \mathrm{C}$ followed by treatment with $\mathrm{BF}_{3} \cdot \mathrm{OEt}_{2}$ afforded $\mathbf{1 3}$ in $47 \%$ yield.

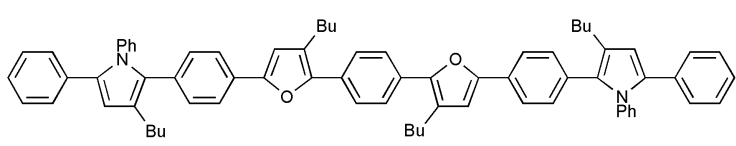

13

Incorporation of double and/or triple bonds into this oligoaryl system is also feasible by combining Heck ${ }^{10}$ or Sonogashira ${ }^{11}$ reaction with the furan annulation protocol. Thus, Heck reaction (10 mol\% $\mathrm{Pd}(\mathrm{OAc})_{2}, 15 \mathrm{~mol} \% \mathrm{Ph}_{3} \mathrm{P}$ and $\mathrm{K}_{2} \mathrm{CO}_{3}$ in $\mathrm{CH}_{3} \mathrm{CN}, 48$ h) of the divinylpentaaryl 12a with excess $\mathbf{4 b}$ afforded 14 in $72 \%$ yield. In a manner similar to that described above, annulation of 14 with $3 \mathrm{~b}$ gave 16 in $62 \%$ yield.

Treatment of 11a with $\mathbf{4 b}$ under Sonogashira conditions (5 mol\% of $\mathrm{PdCl}_{2}\left(\mathrm{PPh}_{3}\right)_{2} 10 \mathrm{~mol} \%$ of $\mathrm{CuI}$ and $\mathrm{Et}_{3} \mathrm{~N}$ in acetonitrile) gave $78 \%$ yield of dialdehyde $\mathbf{1 5}$. Annulation of $\mathbf{1 5}$ with 3c under our usual conditions yielded $17(45 \%)$. Similarly, 18 was obtained in $48 \%$ yield from the reaction of $\mathbf{1 5}$ with $\mathbf{3 b}$. Since 16-18 contain double or triple bonds at the terminal phenyl rings, further transformation by repeating the same procedures would lead to higher homologues.

Electrochemical studies showed that teraryl $\mathbf{5}$ exhibited a reversible one-electron redox process whereas pentamer $\mathbf{6 a}$ showed a reversible two-electron redox process. Slight decomposition was observed when $\mathbf{6 b}$ and $\mathbf{6 c}$ were subjected to twoelectron oxidation. The first oxidation potentials for oligoaryls are summarized in Table 1. As expected, the first oxidation potential of the oligoaryls decreases with increasing conjugation length. Relatively speaking, substrates containing double or triple bonds (e.g. 14-18) were less stable towards electrochemical oxidation. The absorption, fluorescence data and fluorescent quantum yields are also provided in Table 1. As expected, $\lambda_{\max }$ and $\lambda_{\mathrm{em}}$ increase with the increasing conjugation length and reach saturation at the nonamer $(\mathbf{6 b})$ stage. The

Table 1 Photophysical and electrochemical properties of oligoaryls.

\begin{tabular}{lllll}
\hline Compd. & $E_{1 / 2}{ }^{a / \mathrm{eV}}$ & $\lambda_{\max }^{b / \mathrm{nm}}$ & $\lambda_{\mathrm{em}}{ }^{b / \mathrm{nm}}$ & $\Phi_{\mathrm{f}}$ \\
\hline $\mathbf{5}$ & 0.92 & 364 & 402,423 & 0.74 \\
$\mathbf{6 a}$ & 0.57 & 398 & 454,482 & 0.74 \\
$\mathbf{6 b}$ & 0.31 & 418 & 474,480 & 0.53 \\
$\mathbf{6 c}$ & 0.20 & 422 & 474,499 & 0.42 \\
$\mathbf{1 3}$ & 0.41 & 361,395 & $451,485,523$ & 0.79 \\
$\mathbf{1 4}$ & 0.37 & 344,407 & 484 & 0.58 \\
$\mathbf{1 5}$ & 0.40 & 348,409 & 494 & 0.59 \\
$\mathbf{1 6}$ & 0.18 & 422 & 497 & 0.73 \\
$\mathbf{1 7}$ & 0.33 & 397 & 491 & 0.48 \\
$\mathbf{1 8}$ & 0.28 & 402 & 494 & 0.63
\end{tabular}

${ }^{a}$ The first oxidation potential vs. ferrocene/ferrocenium ion. ${ }^{b}$ Measured in $\mathrm{CHCl}_{3}$ solution. presence of pyrrole rings resulted in a red shift of the emission.
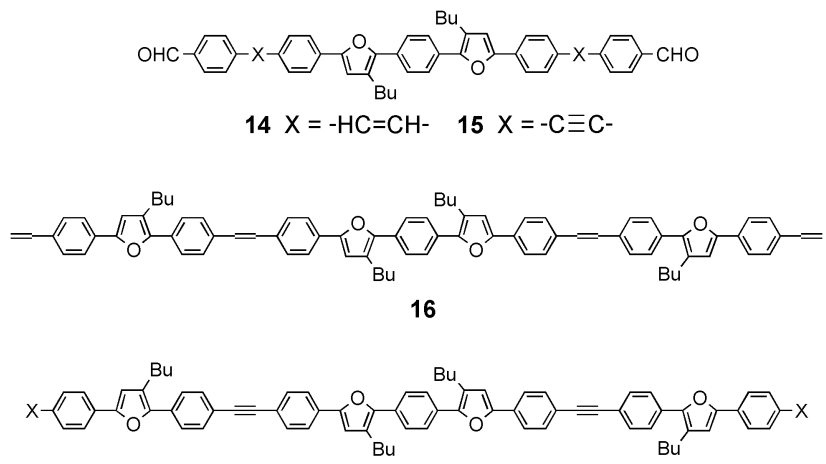

$17 \mathrm{X}=\mathrm{TMSC} \equiv \mathrm{C} \quad 18 \mathrm{X}=\mathrm{H}_{2} \mathrm{C}=\mathrm{CH}$

In summary, we have demonstrated a new route for the synthesis of a variety of alternating benzene-furan oligoaryls up to $5 \mathrm{~nm}$ in length. Further extension by using the same strategy will be feasible leading to the synthesis of molecular wires of well-defined conjugation lengths.

We thank the Ministry of Education and the National Science Council of the Republic of China for financial support.

\section{Notes and references}

$\ddagger$ All new compounds gave satisfactory spectroscopic and analytical data. The details are described in the ESI. ${ }^{\dagger}$

1 (a) Electronic Materials: The Oligomer Approach, ed. K. Müllen and G. Wegner, Wiley-VCH, Weinheim, 1998; (b) J. M. Tour, Acc. Chem. Res., 2000, 33, 791.

2 For a review see: A. Gandini and M. N. Belgacem, Prog. Polym. Sci., 1997, 22, 1203 and references therein.

3 H. Saadeh, T. Goodson, III and L. Yu, Macromolecules, 1997, 30, 4608.

4 (a) R. E. Niziurski-Mann and M. P. Cava, Adv. Mater., 1993, 5, 547; (b) R. E. Niziurski-Mann, C. Scordilis-Kelley, T.-L. Liu, M. P. Cava and R. T. Carlin, J. Am. Chem. Soc., 1993, 115, 887; (c) A. Hucke and M. P Cava, J. Org. Chem., 1998, 63, 7413; (d) J. K. Politis, J. C. Nemes and M. D. Curtis, J. Am. Chem. Soc., 2001, 123, 2537; (e) S. M. Pyo, S. I. Kim, T. J. Shim, H. K. Park and M. Ree, Macromolecules, 1998, 31 4777

5 G. Dufresne, J. Bouchard, M. Belletete, G. Durocher and M. Leclerc, Macromolecules, 2000, 33, 8252 .

6 C.-F. Lee, L.-M. Yang, T.-Y. Hwu, A.-S. Feng, J.-C. Tseng and T.-Y. Luh, J. Am. Chem. Soc., 2000, 122, 4992

7 L.-Z. Zhang, C.-W. Chen, C.-F. Lee, C.-C. Wu and T.-Y. Luh, Chem. Commun., 2002, 2336.

8 (a) H. Ikehira, S. Tanimoto and T. Oida, J. Chem. Soc., Perkin Trans 1 , 1984, 1223; (b) A. Krief, B. Kenda and P. Barbeaux, Tetrahedron Lett. 1991, 32, 2509.

9 A. Boudier, L. O. Bromm, M. Lotz and P. Knochel, Angew. Chem., Int. Ed., 2000, 39, 4414.

10 A. de Meijere and S. Bräse, J. Organomet. Chem., 1999, 576, 88

11 K. Sonogashira, Metal-Catalyzed Cross-coupling Reactions, ed. F. Diederich and P. J. Stang, Wiley-VCH, Weiheim, Germany, 1998, pp 203-229. 